\title{
An Equilibrium-Preserving Discretization for the Nonlinear Rosenbluth-Fokker-Planck Operator in Arbitrary Multi-Dimensional Geometry
}

\author{
W. T. Taitano ${ }^{\text {a,* }}$ L. Chacón ${ }^{\mathrm{a}}$, A. N. Simakov ${ }^{\mathrm{a}}$ \\ ${ }^{a}$ Los Alamos National Laboratory, Los Alamos, NM 87545
}

Keywords: Conservative discretization, exact analytical equilibrium preserving, multiple dimensions, Fokker-Planck, Rosenbluth potentials

PACS:

\section{Introduction}

The Fokker-Planck collision operator is an advection-diffusion operator which describe dynamical systems such as weakly coupled plasmas [1,2], photonics in high temperature environment $[3,4]$, biological [5], and even social systems [6]. For plasmas in the continuum, the Fokker-Planck collision operator supports such important physical properties as conservation of number, momentum, and energy, as well as positivity. It also obeys the Boltzmann's H-theorem [7, 8, 9, 10, 11], i.e., the operator increases the system entropy while simultaneously driving the distribution function towards a Maxwellian. In the discrete, when these properties are not ensured, numerical simulations can either fail catastrophically or suffer from significant numerical pollution [12,13]. There is strong emphasis in the literature on developing numerical techniques to solve the Fokker-Planck equation while preserving these properties $[14,15,16,17,18,19,20,21,12,13,22,23,24]$. In this short note, we focus on the analytical equilibrium preserving property, meaning that the Fokker-Planck collision operator vanishes when acting on an analytical Maxwellian distribution function. The equilibrium preservation property is especially important, for example, when one is attempting to capture subtle transport physics. Since transport arises from small $\mathscr{O}(\varepsilon)$ corrections to the equilibrium [25] (where $\varepsilon$ is a small expansion parameter), numerical truncation error present in the equilibrium solution may dominate, overwhelming transport dynamics.

Chang and Cooper [15] first proposed a discrete equilibrium preservation scheme for the Fokker-Planck operator in a 1D isotropic linear system. Larsen et al. [14] developed an equilibrium preserving scheme for Compton scattering in 1D by using the analytical expression for the transport coefficients. Buet and Le Thanh $[19,20]$ developed a mass and energy conserving, positivity and equilibrium preserving scheme in the Landau (integral) formulation for a 1D isotropic Fokker-Planck operator by leveraging the natural (integral) structure of the Fokker-Planck collision operator. All of the approaches above were demonstrated only in one velocity-(energy) space dimension. In a more recent study by Yoon and Chang [26, 27], the authors discuss an extension of the original Chang-and-Cooper discretization and Buet-and-Le Thanh formulation to a two-dimensional cylindrical velocity-space finite-difference representation for the Landau form of the Fokker-Planck operator. However, its generalization to the Rosenbluth form, which can be numerically implemented in an optimal fashion [12], $O(N)$ with $N$ the number of degrees of freedom in velocity space, is not straight forward.

In this study, we present a discretization approach that exactly preserves the analytical equilibrium for

\footnotetext{
${ }^{*}$ Corresponding author

Email address: taitano@lanl.gov (W. T. Taitano)
} 
a nonlinear Fokker-Planck advection-diffusion operator in arbitrary multi-dimensional velocity space. We remark that our approach, although similar in goal (preservation of the analytical equilibrium), is distinctly different from other approaches discussed in references [15, 19, 20, 14]. Reference [15] proposes a discretization to ensure the analytical Maxwellian for a linear Fokker-Planck equation. In reference [14], both a linear and nonlinear forms of Fokker-Planck equation were considered for a Compton and inverse Compton scattering. An analytical expression for the transport coefficients was required to ensure the discrete equilibrium preserving property. Finally in reference [19], a nonlinear, Landau (integral) form of the Fokker-Planck operator was considered for general coefficients with strict conservation in mass and energy, but a nonlinear solve was required to compute the equilibrium preserving weights. In addition, all of these approaches were developed in 1D (typically energy space). In contrast, our approach (i) can be applied to either the Rosenbluth or Landau formulation, (ii) can be used for arbitrary coefficients, (iii) can use an arbitrary discretization stencil, (iv) can be applied in arbitrary dimensions and geometry, and (v) can be trivially built on top of the conservation strategies discussed in references $[12,13]$. The equilibrium-preserving approach proposed here is complementary to an asymptotic-preserving discretization of the Fokker-Planck equation: while the latter ensures that the solution in equilibrium approximates a Maxwellian, the former eliminates the numerical error of the equilibrium solution, rendering analytically exact.

Herein, we discuss the methodology in the context of weakly coupled plasmas for a Rosenbluth-FokkerPlanck collision operator. We demonstrate through numerical examples that the approach preserves the exact analytical equilibrium. The rest of the manuscript is organized as follows. In Section 2, we discuss the Fokker-Planck operator and some of its properties in the continuum. In Section 3, we discuss our discretization scheme that preserves the analytical equilibrium. In Sections 4 and 5, we provide implementation details for the application to weakly coupled plasmas. In Section 6, we present key numerical results. Finally, we present our conclusions in Section 7.

\section{Rosenbluth-Fokker-Planck Equation}

In spatially homogeneous systems, the Fokker-Planck equation for a time dependent velocity-distribution function, $f(\vec{v}, t)$, can be written as an advection-diffusion equation:

$$
\partial_{t} f=\nabla_{v} \cdot\left[\vec{J}_{D}-\vec{J}_{A}\right]
$$

where $\vec{J}_{D}=\stackrel{\leftrightarrow}{D} \cdot \nabla_{v} f, \vec{J}_{A}=\vec{A} f, \nabla_{v}=\frac{\partial}{\partial \vec{v}}, \overleftrightarrow{D}$ is the diffusion tensor, and $\vec{A}$ is the friction vector. In general, the coefficients $\stackrel{\leftrightarrow}{D}$ and $\vec{A}$ are functions of the solution, $f$. For weakly coupled plasmas [1,2], the coefficients can be evaluated in terms of the Rosenbluth potentials $G$ and $H$ as

$$
\stackrel{\leftrightarrow}{D}=\nabla_{v} \nabla_{v} G, \vec{A}=\nabla_{v} H
$$

where the Rosenbluth potentials, in turn, can be evaluated by inverting the coupled Poisson equations

$$
\nabla_{v}^{2} H=-8 \pi f, \nabla_{v}^{2} G=H .
$$

The Fokker-Planck operator [the right-hand side of equation (2.1)] conserves total particle number, momentum, and energy of the system [2]. The equilibrium solution of equation (2.1) is a Maxwellian, i.e.

$$
\vec{J}_{M}=\vec{J}_{D, M}-\vec{J}_{A, M}=\overleftrightarrow{D}_{M} \cdot \nabla_{v} f_{M}-\vec{A}_{M} f_{M}=0
$$

with

$$
f_{M}=\frac{n}{(2 \pi T / m)^{3 / 2}} \mathrm{e}^{-\frac{m}{2 T}|\vec{v}-\vec{u}|^{2}}
$$


with mass, $m$, number density, $n$, drift velocity, $\vec{u}$, and temperature, $T$. The equilibrium constraint implies that

$$
\vec{J}_{M}=0 \Rightarrow \vec{J}_{M} \cdot \vec{l}=J_{M}^{l}=0
$$

on any surface, with a surface normal vector, $\vec{l}$. In order for the Maxwellian equilibrium to be satisfied numerically, the continuum constraint (2.6) must be enforced in the discrete.

\section{Definition of equilibrium constraint coefficient}

In general, an arbitrary discretization of the Fokker-Planck fluxes, $\vec{J}_{A}$ and $\vec{J}_{D}$, will only preserve the equilibrium to some truncation error $\mathscr{O}\left(\Delta v^{\beta}\right)$,

$$
\widehat{J}_{M}^{t}=\mathscr{O}\left(\Delta v^{\beta}\right)
$$

where the over hat denotes a discrete quantity and $\beta$ denotes the order of the discretization. In order to satisfy the constraint (2.6) exactly, we introduce constraint coefficients, $\alpha_{D}$ and $\alpha_{A}$, to modify the discrete Fokker-Planck flux as:

$$
\widehat{J}_{M}^{*, l}=\widehat{J}_{D, M}^{*, l}-\widehat{J}_{A, M}^{*, l}=\sum_{j=1}^{d}\left(1+\alpha_{D}^{j}\right) \widehat{D}_{M}^{l, j} \partial_{j} f_{M}-\left(1+\alpha_{A}\right) \widehat{A}_{M}^{l} f_{M}
$$

where $d$ is the velocity-space dimension, $\widehat{D}_{M}^{l, j}$ denotes the $j$ component of the discrete-Maxwellian tensordiffusion matrix, $\widehat{A}_{M}^{l}$ is the discrete-Maxwellian-friction-coefficient, and $f_{M}$ is the Maxwellian distribution function. We remark that rather than choosing a single constraint coefficient to absorb the truncation error, we introduce several constraint coefficients that affect diffusion and friction fluxes in a balanced manner to distribute the truncation error across collisional fluxes. These constraint coefficients are chosen to enforce equation (2.6) discretely as follows.

Since there are more constraint coefficients than constraint equations $(d+1$ versus 1$)$, the $\alpha$ coefficients are found from a constrained-minimization procedure of the following objective function at a given cell face, $l$ :

$$
F_{l}=\frac{\sum_{j}\left(\alpha_{D}^{j}\right)^{2}+\alpha_{A}^{2}}{2}-\lambda \widehat{J}_{M}^{*, l},
$$

where $\lambda$ is a Lagrange multiplier. The solution for $\alpha$ is found analytically by a least-squares procedure, which relates the $\alpha$ coefficients to numerical derivatives of the Maxwellian, $f_{M}$, at cell faces. This procedure provides the smallest-magnitude coefficients, $\alpha$, compatible with the desired equilibrium constraint. To avoid artifacts away from equilibrium, the correction factor $\alpha$ are only applied when the solution is close enough to equilibrium. We employ the following scheme:

$$
\widehat{J}_{D}^{*, l}=\sum_{j}\left[1+(1-\theta) \alpha_{D}^{j}\right] \widehat{D}^{l, j} \widehat{\partial}_{j} \widehat{f}, \widehat{J}_{A}^{*, l}=\left[1+(1-\theta) \alpha_{A}\right] \widehat{A}^{l} \widehat{f},
$$

where

$$
\theta=\mathrm{a}\{\tanh [2(\chi-2-\gamma)]-\tanh [2(-2-\gamma)]\}
$$

and 


$$
\begin{gathered}
\chi=\frac{\left|v^{2}\left(f-f_{M}\right)\right|_{2}}{\int d^{3} v\left(v^{2} f_{M}\right)} \\
\gamma=\frac{\Delta v}{v_{t h}} \\
a=\frac{1}{1-\tanh [2(-2-\gamma)]} .
\end{gathered}
$$

Here, $\theta$ is a blending factor that detects the closeness of solution to a Maxwellian, $\chi$ is the measure of error from the equilibrium ( $v^{2}$ weighting is chosen to detect tail equilibration) and $\gamma$ measures the coarseness of the grid, where $\Delta v$ is the grid resolution and $v_{t h}=\sqrt{2 T / m}$ is the thermal velocity of the species, which is providing the transport coefficients. The blending factor ensures that if the solution is far from a Maxwellian (e.g. beam equilibrating on a background), the method is not applied $\left(\lim _{\chi \rightarrow \infty} \theta=1\right.$ and $\left.\widehat{J}^{*, l}=\widehat{J}^{l}\right)$. When active, the constraint coefficients, $\alpha$, enslave the $\mathscr{O}\left(\Delta v^{\beta}\right)$ error in the constraint (2.6) into the definition of the Fokker-Planck fluxes. As a result, $\alpha=\mathscr{O}\left(\Delta v^{\beta}\right)$ and $\lim _{\Delta v \rightarrow 0} \alpha=0$, ensuring consistency of the discretization.

\section{Discretization of the FP collision operator}

In order to solve the minimization problem for $\alpha$, one needs to numerically evaluate $\overleftrightarrow{D}_{M}$ and $\vec{A}_{M}$, the equilibrium transport coefficients. They can be obtained from equation (2.2), and the equilibriumRosenbluth potentials,

$$
H_{M}=n\left[\operatorname{erf}(x) / v^{\prime}\right], G_{M}=n v^{\prime}\left[\left(1+\frac{1}{2 x^{2}}\right) \operatorname{erf}(x)+\frac{e^{-x^{2}}}{\sqrt{\pi} x}\right]
$$

with $x=v^{\prime} / \sqrt{2 T / m}$ and $v^{\prime}=|\vec{v}-\vec{u}|$ and $\operatorname{erf}(x)$ the error function We also need to evaluate the surface flux. This requires a stencil for $f_{M}$, which should be chosen to preserve the positivity of the solution (in this study, SMART [28] and mimetic tensor-diffusion differencing [29] are employed). As a final note, in order to ensure the uniqueness of the solution, the actual discretization stencil of the Fokker-Planck collision operator must be consistent with that used for fluxes in the minimization principle (3.2). To illustrate this idea in a finite difference context, we define a face interpolation of a cell-centered distribution function as:

$$
\widehat{f}_{\text {face }}(\widehat{f}, \widehat{g})=\sum_{i=1}^{N_{g}} \omega_{i}(\widehat{g}) \widehat{f}_{i}
$$

where $N_{g}$ is the number of grid points in the direction of interpolation (assuming a structured grid), $\omega_{i}(\widehat{g})$ is the interpolation weight contribution of $\widehat{f_{i}}$ (according to $\widehat{g}$ ) to the surface flux, and $\sum_{i=1}^{N_{g}} \omega_{i}(\widehat{g})=1$. When the solution is far from equilibrium, the Maxwellian stencil in the minimization principle does not guarantee positivity for arbitrary $f$, and thus the stencil based on the current $f$ is used (i.e., $\widehat{g}=\widehat{f}$ ). We employ the Maxwellian stencil (i.e., $\widehat{g}=f_{M}$ ) only when $f$ is sufficiently close to the equilibrium solution (i.e., when $\frac{\left|\widehat{f}-f_{M}\right|_{2}}{n} \leq 10^{-2}+\frac{\Delta v}{2 v_{t h}}$ in this study).

The goal of our discretization is to ensure that, when $\widehat{f}=f_{M}$, the collision operator evaluates discretely to zero. This in turn, requires the Rosenbluth potentials to evaluate discretely to the Maxwellian counter parts. For this, we consider the following form of the Rosenbluth Poisson equations (2.3):

$$
\widehat{\nabla}_{v}^{2} \delta \widehat{H}=-8 \pi \delta \widehat{f}, \widehat{\nabla}_{v}^{2} \delta \widehat{G}=\delta \widehat{H}
$$


where

$$
\delta \widehat{G}=\widehat{G}-G_{M}, \delta \widehat{H}=\widehat{H}-H_{M}, \delta \widehat{f}=\widehat{f}-f_{M} .
$$

This formulation ensures that, if the solution is a Maxwellian $\left(\widehat{f}=f_{M}\right)$, then $\delta \widehat{H}=0$ and $\delta \widehat{G}=0$ and

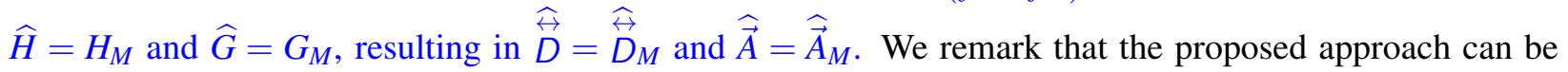
extended to a multiple-grid approach as discussed in reference [13] by coarse-graining and interpolating the potentials between grids rather than re-solving the Poisson equation.

We summarize the key steps of the proposed equilibrium preserving discretization procedure as follows:

1. Evaluate the Maxwellian diffusion and advection fluxes with a positivity-preserving discretization for

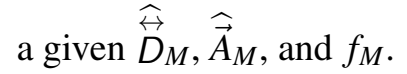

2. Find $\alpha$ coefficients by minimizing the objective function (3.2).

3. Compute the transport coefficients for the current state by solving the coupled Poisson equations for the Rosenbluth potentials (4.3), (4.4) and discretely differentiating them following equation (2.2).

4. Evaluate the diffusion and advection fluxes with a positivity-preserving discretization for a given $\stackrel{\leftrightarrow}{D}$, $\overrightarrow{\widehat{A}}$, and $\widehat{f}$.

5. Modify the fluxes from step 4 according to equation (3.3).

We remark that the method can be applied to a large class of grid-based discretizations where the FokkerPlanck operator is represented in terms of a divergence of a flux (e.g. finite difference, finite volume, and finite element methods). Additionally, since the basic idea relies on modifying the Fokker-Planck fluxes at cell faces, one can extend it to arbitrary geometries and also employ the discrete conservation strategy discussed in references $[12,13]$. The numerical results section, Section 6 , considers a cylindrically symmetric velocity space.

\section{Accounting for discrete quadrature (moments) errors in the equilibrium solution}

The Maxwellian distribution function, $f_{M}$, is given by equation (2.5) for prescribed $n, \vec{u}$, and $T$. In order to guarantee that the analytical equilibrium solution is recovered for a given set of moments, one must also account for numerical-quadrature errors in the moment computation even when $f_{M}$ is analytical. We ensure that discrete moments of the Maxwellian yield the prescribed values of $(n, u, T)$ as follows. The constraints to be satisfied are:

$$
\begin{gathered}
n-\sum_{i} \sum_{j} \sum_{k} \Delta V_{i j k} \frac{n_{M}}{\left(2 \pi T_{M} / m\right)^{3 / 2}} e^{-\frac{m}{2 T_{M}}\left|\vec{v}-\vec{u}_{M}\right|^{2}}=0, \\
\vec{u}-\frac{\sum_{i} \sum_{j} \sum_{k} \Delta V_{i j k} \vec{v} e^{-\frac{m}{2 T_{M}}\left|\vec{v}-\vec{u}_{M}\right|^{2}}}{\sum_{i} \sum_{j} \sum_{k} \Delta V_{i j k} e^{-\frac{m}{2 T_{M}}\left|\vec{v}-\vec{u}_{M}\right|^{2}}}=0, \\
T-\frac{m}{3} \frac{\sum_{i} \sum_{j} \sum_{k} \Delta V_{i j k}\left|\vec{v}-\vec{u}_{M}\right|^{2} e^{-\frac{m}{2 T_{M}}\left|\vec{v}-\vec{u}_{M}\right|^{2}}}{\sum_{i} \sum_{j} \sum_{k} \Delta V_{i j k} e^{-\frac{m}{2 T_{M}}\left|\vec{v}-\vec{u}_{M}\right|^{2}}}=0 .
\end{gathered}
$$

Inputs are $n, \vec{u}, T$. The solution to the above nonlinear system yields numerical values of $n_{M}, u_{M}$, and $T_{M}$ to be used for the Maxwellian distribution function such that prescribed moments $n, \vec{u}, T$ are recovered exactly. Here, $\Delta V_{i j k}=\Delta v_{1, i} \Delta v_{2, j} \Delta v_{3, k}, \vec{u}_{M}=\left[u_{M, 1}, u_{M, 2}, u_{M, 3}\right], \vec{v}=\left[v_{1, i}, v_{2, j}, v_{3, k}\right]$ where $i, j$, and $k$ are the grid indices for the respective directions. We remark that, in order to solve numerically the above nonlinear system, a minimum grid resolution, $\Delta v_{\min } \leq 1.5 v_{t h}$, and sufficiently large v-space domain, $\left|u-L_{v}\right| \geq 2.5 v_{t h}$, $\left(v_{t h}=\sqrt{2 T / m}\right)$, must be provided. When providing an initial guess of $n_{M}=n, u_{M}=u$, and $T_{M}=T$ the Newton's method converges to roundoff in about three iterations. 

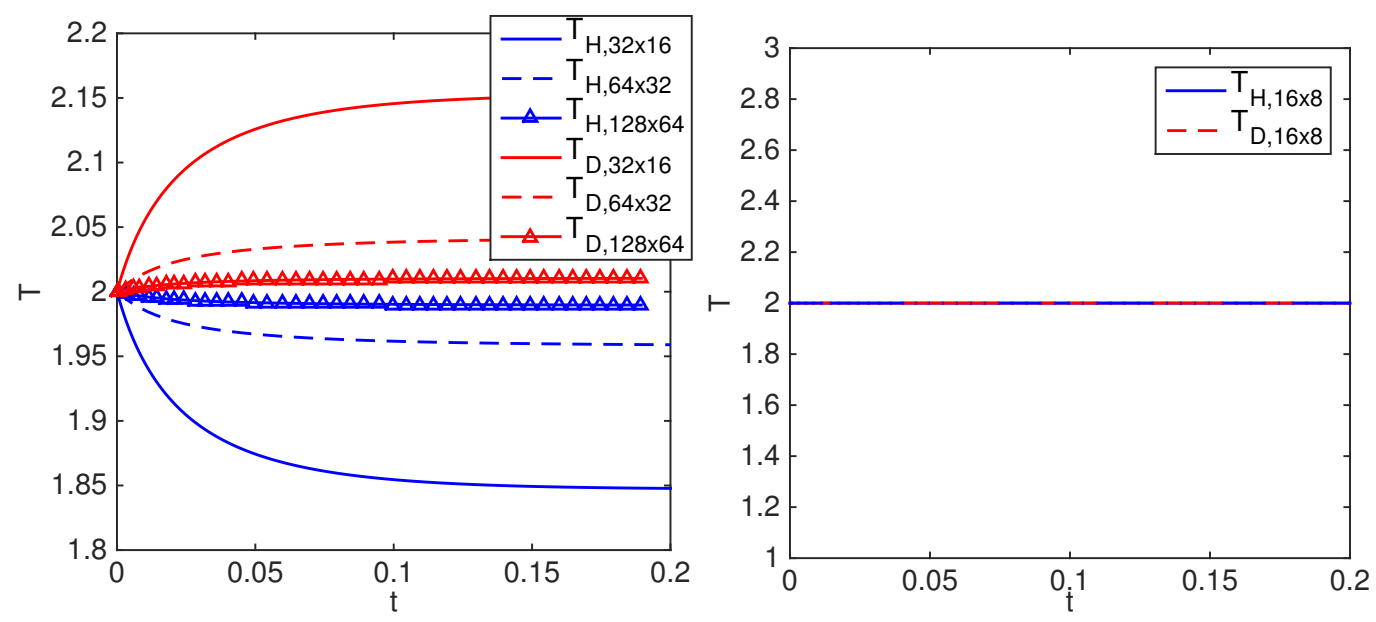

Figure 6.1: Preservation of equilibrium: Comparison of numerical solutions for a standard discretization (left) and equilibriumpreserving discretization (right).

\section{Numerical Results}

We test the new discretization scheme on several problems that highlight key properties of the equilibrium preserving scheme. We employ a conservative finite-volume formulation with a two-dimensional azimuthally symmetric cylindrical-velocity space $\left(v_{\|}, v_{\perp}\right)$. In all problems, unless otherwise stated, the PDF, $f$, is initialized with a Maxwellian,

$$
f=\frac{n_{M}}{\left(2 \pi T_{M} / m\right)^{3 / 2}} \mathrm{e}^{-m \frac{\left(v_{\|}-u_{\|, M}\right)^{2}+v_{\perp}^{2}}{2 T_{M}}},
$$

with prescribed density, $n$, parallel drift velocity, $u_{\|,}$, and temperature, $T$. The moments $n_{M}, u_{\|, M}$, and $T_{M}$ are computed by solving equations (5.1), (5.2), and (5.3). We note that, unless otherwise stated, the numerical simulations were performed with the discrete conservation strategy discussed in reference [12].

\subsection{Preservation of equilibrium}

Consider a quasi-neutral, fully ionized plasma containing proton and deuteron ions in equilibrium. The ion masses are $\left(m_{p}, m_{d}\right)=(1,2)$, densities are $\left(n_{p}, n_{d}\right)=(1,1)$, drift velocities are $\left(u_{\|, p}, u_{\|, d}\right)=(0,0)$, and temperatures are $\left(T_{p}, T_{d}\right)=(1,1)$. The domain considered is $L \equiv L_{\|} \times L_{\perp} \in[-5,5] \times[0,5]$. Physically, this system should remain in equilibrium. In Figure 6.1, we show temperatures of the two ion species as functions of time for different grids with the conservative discretization (left) and with the new equilibriumpreserving scheme (right). With the conservative discretization, owing to truncation errors, the numerical solution deviates from the Maxwellian (even though the mass, momentum, and energy are conserved exactly [12]). The new scheme preserves the correct analytical equilibrium indefinitely.

\subsection{Proton beam slowing down on carbon}

We consider a warm proton beam described by a Maxwellian distribution with $m_{b}=1, n_{b}=1, u_{\|, b}=-2$, $T_{b}=0.2$, slowing down on a static background of carbon ions with $n_{c}=1, u_{c}=0, T_{c}=1$. We neglect the evolution of carbon in this problem. The purpose of this test is 1) to demonstrate that an out-of-equilibrium PDF, $f$, evolves to the analytical equilibrium, determined by the background carbon's drift and temperature and 2) to assess the dynamical convergence of the numerical solution to the analytical equilibrium. We consider the computational domain $L_{v} \equiv L_{\|} \times L_{\perp} \in[-6,6] \times[0,6]$, with a grid of $N_{v}=N_{\|} \times N_{\perp}=32 \times 16$ (coarse on purpose). 

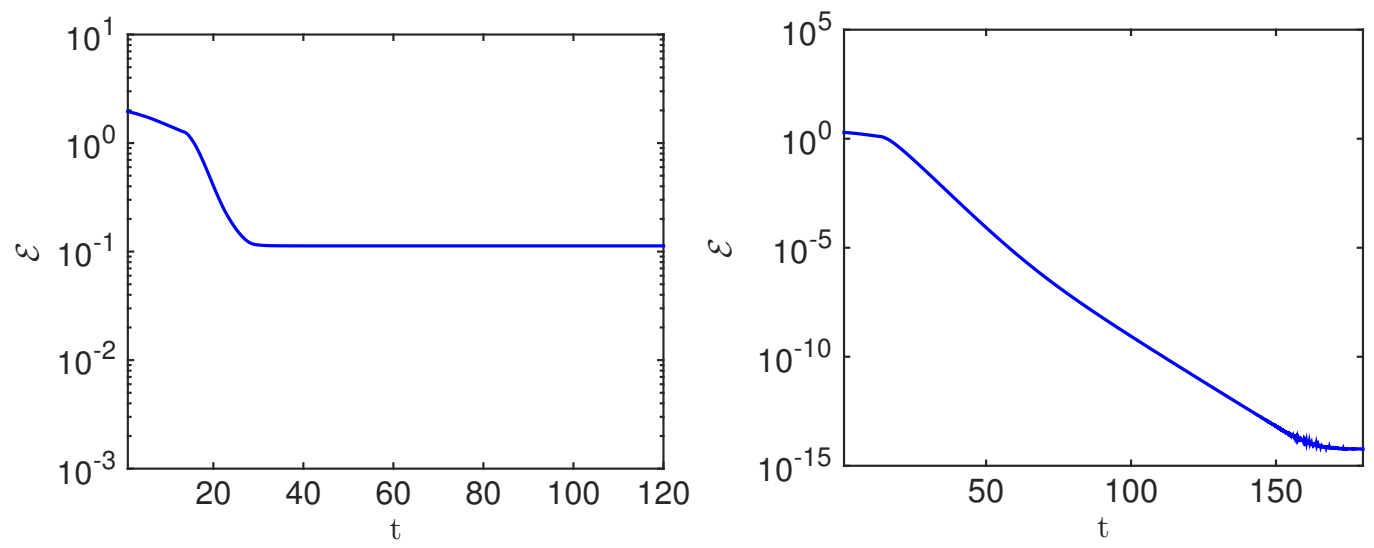

Figure 6.2: Proton beam slowing down on carbon: Comparison in convergence rate of the solution to the analytical equilibrium for a standard discretization (left) and an equilibrium-preserving scheme (right).

Figure 6.2 shows the $L_{2}$ norm of the deviation of the proton-beam distribution function from the analytical equilibrium,

$$
\mathscr{E}=\left|f(t)-f_{M}\left(m_{b}, n_{b}, u_{c}, T_{c}\right)\right|_{2},
$$

as a function of time, for the non-equilibrium-preserving (standard) discretization scheme (left) and the equilibrium-preserving scheme (right). As can be seen, with the standard discretization considered in references $[12,13]$, the solution never reaches the analytical equilibrium, while with the new scheme the solution monotonically converges to the analytical solution (to machine roundoff). Figure 6.3 shows the phase-space transport process (slowing down and parallel/perpendicular diffusion) of the beam due to the background structure. We note once again that the new equilibrium-preserving discretization only determines the quality of the equilibrium, and that it does not alter the physics of the problem.

\subsection{Temperature equilibration of three plasma species}

As a final test, we consider a three-species thermal equilibration case where a mix of proton, deuteron, and carbon ions is initialized with different temperatures. The ion parameters are as follows: $\left(m_{p}, m_{d}, m_{c}\right)=$ $(1,2,12),\left(n_{p}, n_{d}, n_{c}\right)=(1,1,1),\left(u_{\|, p}, u_{\|, d}, u_{\|, c}\right)=(0,0,0),\left(T_{p}, T_{d}, T_{c}\right)=(0.5,1,1.5)$. For the grid, we consider a domain of $L \in[-6,6] \times[0,6]$ with various refinement levels. In Figure 6.4, the time history of the three species temperatures as functions of time is plotted for both the standard discretization and the equilibrium-preserving scheme. As can be seen, without the equilibrium-preserving scheme, the equilibrium departs significantly from the analytical one, requiring a grid of $N=128 \times 64$ to approach the analytical equilibrium solution to within a few-percent error. Total energy is conserved in all cases. With the equilibrium-preserving scheme, even a coarse grid of $32 \times 16$ drives the solution to the exact equilibrium, as expected. We note that for this problem, the combined cost of steps 1, 2, 4, and 5 in Sec. 4 were less than $0.1 \%$ of the cost of inverting the Poisson equations and the collision operator (optimally, as discussed in reference [12]), independently of mesh refinement.

\section{Conclusions}

We have proposed a novel equilibrium-preserving discretization for nonlinear advection-diffusion FokkerPlanck operators in arbitrary multi-dimensional geometries. The central idea of the approach is to introduce a suitable set of constraint coefficients into the definition of the Fokker-Planck fluxes to ensure fluxcancellation in equilibrium. Several numerical examples in cylindrical velocity-space geometry demonstrate 

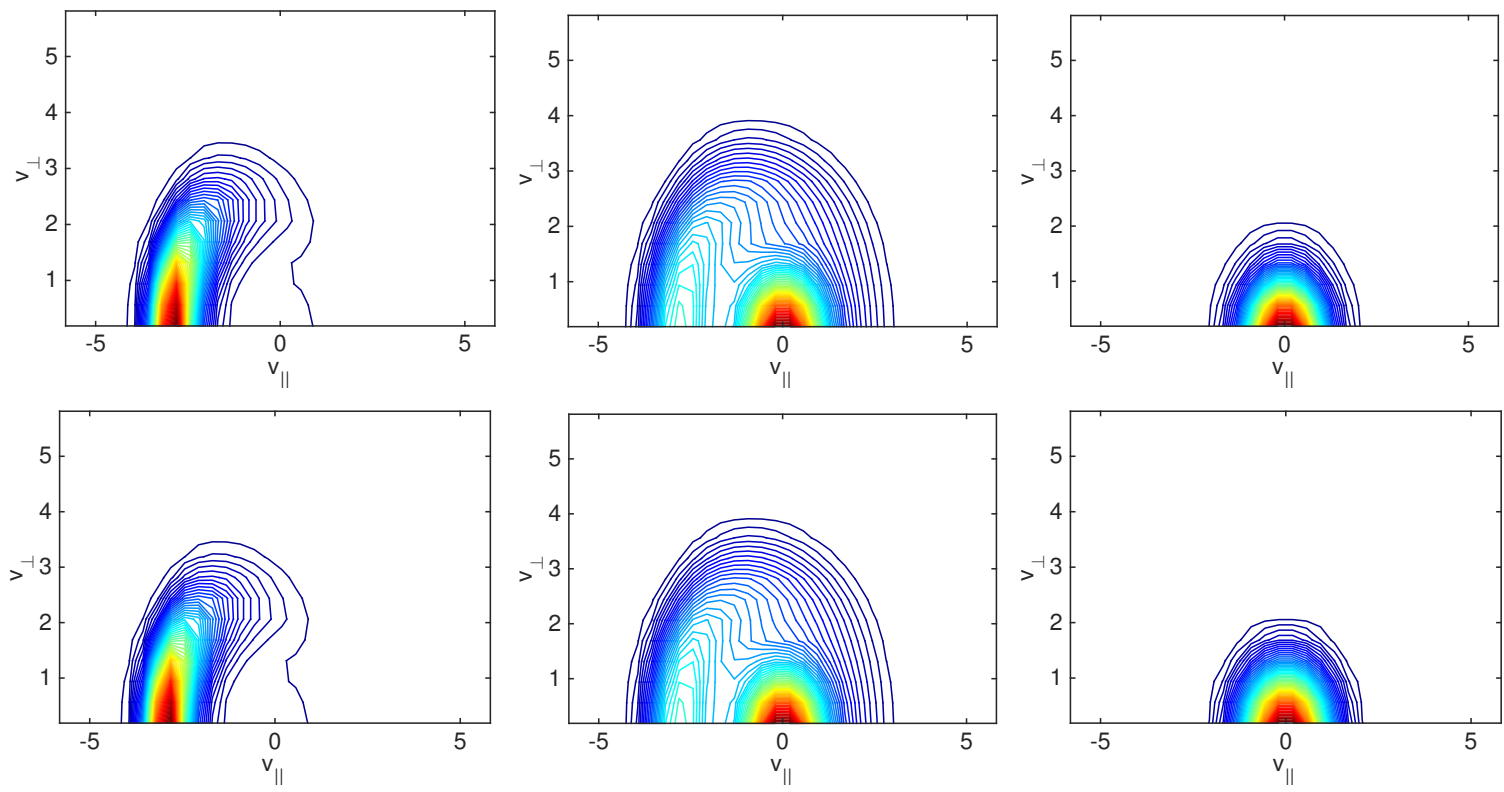

Figure 6.3: Proton beam slowing down on carbon: The proton beam distribution function is shown at various times (from left, $t=6.5,19.5,130.3$ ) for the equilibrium-preserving discretization (top row) and standard discretization (bottom row). No noticeable differences are seen between these studies, except at equilibrium.

the effectiveness of the approach. The proposed approach can be extended to other Fokker-Planck systems and grid-based discretizations as long as 1) the advection-diffusion structure of the operator is maintained and 2) the equilibrium solution is known for such a system.

\section{Acknowledgments}

We would like to thank the anonymous referees, whose comments and suggestions were used to improve the quality and clarity of this presentation. This work was sponsored by the Metropolis Postdoctoral Fellowship, the LDRD office, and the Thermonuclear Burn Initiative of the Advanced Simulation and Computing Program at Los Alamos National Laboratory. This work was performed under the auspices of the National Nuclear Security Administration of the U.S. Department of Energy at Los Alamos National Laboratory, managed by LANS, LLC under contract DE-AC52-06NA25396.

[1] M. N. Rosenbluth, W. M. Macdonald, and D. L. Judd, "Fokker-Planck equation for an inverse-square force," Phys. Rev., vol. 107, no. 1, pp. 1-6, 1957.

[2] L. D. Landau, "The kinetic equation in the case of Coulomb interaction," Phys. Zs. Sov. Union, vol. 10, pp. 154-164, 1936.

[3] A. S. Kompaneets, "The establishment of thermal equilibrium between quanta and electrons," Sov. Phys., JETP, vol. 4, no. 5, p. 730, 1957.

[4] Y. B. Zel'dovich, E. V. Levich, and R. A. Syunyaev, "Stimulated compton interaction between Maxwellian electrons and spectrally narrow radiation," Sov. Phys., JETP, vol. 35, no. 4, pp. 733-740, 1972.

[5] P. H. Chavanis, "Nonlinear mean field Fokker-Planck equations. Application to the chemotaxis of biological population,” p. arXiv:0709.1829v2, 2008. 

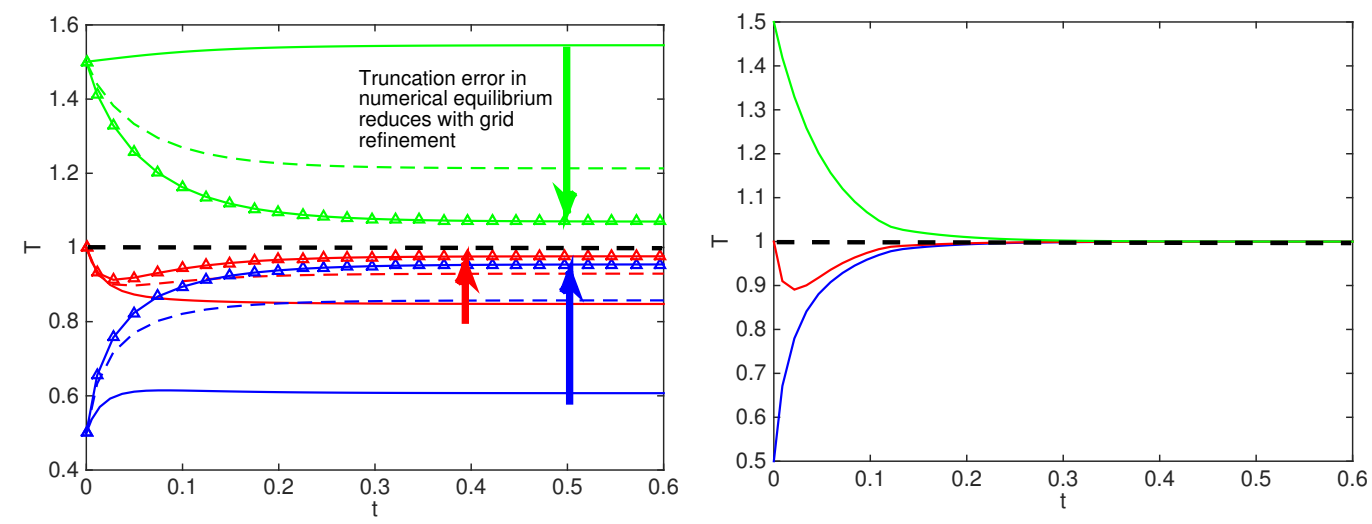

Figure 6.4: Temperature equilibration of three species: Comparison between a standard discretization (left) and an equilibriumpreserving scheme (right). The black dashed line denotes the analytical equilibrium temperature value and the blue, red, and green lines denotes the proton, deuteron, and carbon temperatures, respectively. For the standard discretization plot, solid, dashed, and triangle-marked lines pertain to $32 \times 16,64 \times 32$, and $128 \times 64$ grids, respectively. The results for equilibrium-preserving scheme were obtained on a $32 \times 16$ grid.

[6] P. Degond, J. G. Liu, S. Merino-Aceituno, and T. Tardiveau, "Continuum dynamics of the intention field under weakly cohesive social interactions," p. arXiv:1607.06372v1, 2016.

[7] A. A. Arsen'ev and O. E. Buryac, "On the connection between a solution of the Boltzmann equation and a solution of the Landau-Fokker-Planck equation," USSR Comput. Maths math. Phys., vol. 17, pp. 241-246, 1991.

[8] L. Desvillettes, "On asymptotics of the Boltzmann equation when the collisions become grazing," Trans. Theory and Stat. Phys., vol. 21, no. 3, pp. 259-276, 1992.

[9] P. Degond and B. Lucquin-Desreux, "The Fokker-Planck asymptotics of the Boltzmann collision operator in the Coulomb case," Math. Models Meth. Appl. Sci., vol. 2, no. 2, pp. 167-182, 1992.

[10] T. Goudon, "On Boltzmann equations and Fokker-Planck asymptotics: Influence of grazing collisions," J. Stat. Phys., vol. 89, no. 3/4, pp. 751-776, 1997.

[11] R. D. Hazeltine and J. D. Meiss, Plasma Confinement. Redwood City, CA: Addison-Wesly Publishing Company, 1991.

[12] W. T. Taitano, L. Chacón, A. N. Simakov, and K. Molvig, "A mass, momentum, and energy conserving, fully implicit, scalable algorithm for the multi-dimensional, multi-species Rosenbluth-Fokker-Planck equation," J. Comput. Phys., vol. 297, pp. 357-380, 2015.

[13] W. T. Taitano, L. Chacón, and A. N. Simakov, "An adaptive, conservative 0D-2V multispecies Rosenbluth-Fokker-Planck solver for arbitrarily disparate mass and temperature regimes," J. Comput. Phys., vol. 318, pp. 391-420, 2016.

[14] E. W. Larsen, C. D. Levermore, G. Pomraning, and J. G. Sanderson, "Discretization methods for onedimensional Fokker-Planck operators," J. Comput. Phys., vol. 61, pp. 359-390, 1985.

[15] J. S. Chang and G. Cooper, "A practical difference scheme for Fokker-Planck equations," J. Comput. Phys., vol. 6, pp. 1-16, 1970. 
[16] P. Degond and B. Lucquin-Desreux, "An entropy scheme for the Fokker-Planck collision operator of plasma kinetic theory," Numerische Mathematik, vol. 68, pp. 239-262, 1994.

[17] F. Filbet and S. Jin, "A class of asymptotic-preserving schemes for kinetic equations and related problems with stiff sources," J. Comput. Phys., vol. 229, pp. 7625-7648, 2010.

[18] F. Filbet and L. Pareschi, "A numerical method for the accurate solution of the Fokker-Planck-Landau equation in the nonhomogeneous case," J. Comput. Phys., vol. 179, pp. 1-26, 2002.

[19] C. Buet and K. C. Le-Thanh, "About positive, energy conservative and equilibrium state preserving schemes for the isotropic Fokker-Planck-Landau equation,” HAL-00092543, 2006.

[20] C. Buet and K. C. Le-Thanh, "Positive, conservative, equilibrium state preserving and implicit difference schemes for the isotropic Fokker-Planck-Landau equation," HAL-00142408, 2007.

[21] L. Chacón, D. C. Barnes, D. A. Knoll, and G. H. Miley, "An implicit energy-conservative 2D FokkerPlanck algorithm: I- Difference scheme,” J. Comput. Phys., vol. 157, no. 2, pp. 618-653, 2000.

[22] M. S. Pekker and V. N. Khudik, "Conservative finite-difference schemes for the Fokker-Planck equation," USSR Comput. Maths. math. Phys., vol. 24, no. 3, pp. 206-210, 1984.

[23] E. M. Epperlein, "Implicit and conservative difference scheme for the Fokker-Planck equation," $J$. Comput. Phys., vol. 112, pp. 291-297, 1994.

[24] A. B. Langdon, "Conservative differencing of the electron fokker-planck transport equation," in Centre Européen de Calcul Atomique et Moléculaire (CECAM) Workshop on The Flux Limiter and Heat Flow Instabilities in Laser-Fusion Plasmas, (Orsay, France), p. 69, Université Paris Sud, 14 Sept.-2 Oct. 1981.

[25] S. I. Braginskii, "Transport processes in a plasma," in Reviews of Plasma Physics (M. A. Leontovich, ed.), vol. 1, pp. 205-311, New York: Consultants Bureau, 1965.

[26] E. S. Yoon and C. S. Chang, "A Fokker-Planck-Landau collision equation solver on two-dimensional velocity grid and its application to particle-in-cell simulation," Phys. Plasmas, vol. 21, p. 032503, 2014.

[27] E. S. Yoon and C. S. Chang, "Erratum:" A Fokker-Planck-Landau collision equation solver on twodimensional velocity grid and its application to particle-in-cell simulation"," Phys. Plasmas, vol. 21, p. 039905, 2014.

[28] P. H. Gaskell and A. K. C. Lau, "Curvature-compensated convective transport: SMART, a new boundedness-preserving transport algorithm," Intern. Journ. Num. Meth. in Fluids, vol. 8, pp. 617$641,1988$.

[29] K. Lipnikov, D. Svyatskiy, and Y. Vassilevski, "Minimial stencil finite volume scheme with the discrete maximum principle," Russ. J. Numer. Anal. Math. Modelling, vol. 27, no. 4, pp. 369-385, 2012. 